\title{
Diagetic vs. Non-Diagetic Game Displays
}

\author{
Margaree Peacocke, Robert J. Teather, Jacques Carette \\ Dept. of Computing and Software \\ McMaster University \\ Hamilton, Canada
}

\begin{abstract}
The method used to display game information, either overlay or in-game, in first-person shooters, likely has a measurable impact on player performance. Quantitative research is needed in this area, as game designers are pushing for heads-up displays to be as minimal as possible. This is especially important for the smaller screens (such as tablets and even cellular devices), which are now being targeted by this genre. Quantifying the effect of information displays across different screen sizes (small as well as traditional monitors and TVs) will allow for interfaces which improve the user's performance and gameplay experience.
\end{abstract}

Keywords-Heads-up displays, diagetic user interfaces, games.

\section{INTRODUCTION}

A typical design objective of first-person shooter (FPS) games is to create the most immersive experience possible for players. One option to potentially enhance immersion is to replace common heads-up display (HUD) elements (e.g., health bars, ammo counters, etc.) with in-game methods of communicating the same information. However, there is evidence that player immersion is more strongly affected by overall game coherence than by minimizing the number of HUD elements [1]. Similarly, little work has explored the most effective means of communicating gameplay information to players. We thus explore current trends in FPS UI design, primarily comparing diagetic (immersive elements that exist in the game world) and standard (HUD elements) in terms of both their effects on player performance and experience.

A major advantage of diagetic UIs is their relatively small "footprint" on the display. Consider HUD-heavy FPS games on tablets and cell phones: the display may be crowded with HUD elements as depicted in Fig. 1. Similarly, touch displays often require on-screen controls which also take up a large amount of space on a comparatively small display. In contrast, games using diagetic UI elements may inherently scale better to smaller displays as such UI elements do not require additional display space. Our work focuses on determining the best ways to display health, current weapon, remaining ammunition, and navigational aids on varying display sizes. The objective is to provide developers recommendations for porting games from large to small displays.

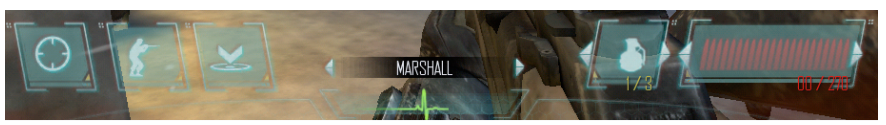

Fig. 1. The Call of Duty: Strike Team HUD on iOS contains controls, health indicator, special weapon ammo count, and regular weapon ammo count.

\section{RELATED WORK}

Previous work compared player immersion between HUDs and diagetic UIs. Fagerholt and Lorentzon [1] describe three layers of the game interface: the game itself, the filter, and the overlay. They studied player preference for health and navigation aids and found that game coherence - when the game theme is consistent with the HUD theme - is a stronger determinant of immersion than display type. Llanos and Jorgensen [2] report on a qualitative study of player preference comparing diagetic and HUD-based UIs. While participants liked the aesthetic appeal of diagetic interfaces, they preferred to have information communicated clearly. A similar study indicated that HUD-based UIs were not considered disruptive. [3] Other work investigated visualization techniques for HUD overlay elements [4]. This included a visual analysis of Valve Software's well-known Half Life 2 to assess techniques used to display quantitative information in the game.

\section{CurRent GAMES ANALysis}

Like previous work [1], we analysed popular FPS games including EA's Call of Duty: Strike Team, Call of Duty: Black Ops, Call of Duty: Ghost, Ubisoft's Tom Clacy's Rainbow Six: Vegas, Bioware's Mass Effect 3, Mass Effect Infiltrator and EA's Dead Space. The games are available on multiple platforms, including PC and tablet. Our analysis involved playing the games, watching gameplay videos, and reading reviews. We identified four items common to all games: health, current weapon, ammunition count, and navigation aid. We report the most common methods to display each item.

\section{A. Health}

The most common methods to display health were health bars and visual filters. The health bar is presented on the HUD, while visual filters take several forms (e.g., blood spatter when the player is hit). The visual filter is a meta-perception [1], a semi-diagetic element considered to be a part of the game's fiction, and thus may be more immersive.

\section{B. Current Weapon}

Current weapon is usually indicated by showing the weapon in front of the character. This is a diagetic display [1] the weapon is part of the game's fiction and present in the game space. This may be used simultaneously with a HUD display, e.g., a weapon icon and/or name (see Fig. 2). These are displayed constantly or as appropriate (e.g., during battle). 


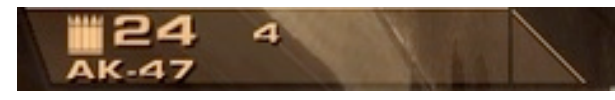

Fig. 2. Tom Clancy's Rainbow Six: Vegas constantly displays weapon names

\section{Ammunition Count}

Ammunition count is almost always displayed on the HUD. This is likely because of the comparative difficulty in designing a diagetic ammo display that fits the game theme. Common methods include an icon bar, regular bar, numerical indicator, textual indicator, or combinations of these (see Fig. 3) Note that some diagetic options exist: for example, EA's Dead Space shows a numerical indicator above the weapon, while Metro 2033 by 4A Games visualizes bullets remaining in the gun.

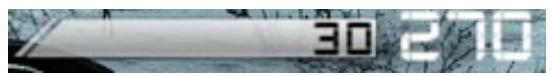

Fig. 3. Call of Duty: Ghosts uses a numerical indicator and bar

\section{Navigation Aids}

Navigation aids are commonly displayed as overlays. The two most common methods are mini-maps (i.e., a HUD element in the corner of the screen), and directional arrows displayed within the gamespace but which are not a part of the game's fiction. Note that there is some debate over the necessity of navigation aids. Many gamers argue that such aids limit the exploratory nature of games.

\section{RANKING OF UI ELEMENTS}

Our long term goal is to rank display options for health, current weapon, ammunition count, and navigation aid for several screen sizes (e.g., TV and mobile). This includes assessment of which display options are interchangeable. This will help determine if cluttered HUD overlays could be (partly) replaced with in-game diagetic displays instead. We thus propose an experiment to determine the relative performance benefits of each display option.

\section{PROPOSED EXPERIMENT}

We plan to conduct a user study comparing diagetic and non-diagetic displays for health, current weapon, ammunition count, and navigational aids. A primary objective is to assess each as a balance of speed, precision, and user preference. This study will include three phases on three different platforms: A computer (with a large TV), a tablet, and a cell phone.

We will develop a FPS game specifically for the study. This offers considerably greater experimental control [5] and will avoid bias participants may have towards existing games [1]. The game will consist of four tasks, each intended to isolate one of the four display types described above. Any other displays will not matter during each task. The software will record participant performance in each task under the different display conditions. Upon completion of the experiment, participants will be surveyed for their preferred display methods. The tasks and conditions are described below.

\section{A. Health Display Evaluation}

Participants will fight difficult enemies until their health reaches a critical level, at which point they will move to safety to regenerate health. The use of health bar (non-diagetic), health bar (diagetic, as in Dead Space), and a blood filter as health indicators will be evaluated. Time to notice critical health, and how often they fail the task will be recorded.

\section{B. Current Weapon Display Evaluation}

Participants will play a level with several areas, where a different weapon is required in each. They will need to be able to quickly switch to and identify the correct weapon. Having the weapon displayed in front of the character (diagetic), a weapon icon (non-diagetic), and weapon name (non-diagetic) will be evaluated. Time to identify the correct weapon, and frequency of choosing an incorrect weapon will be recorded.

\section{Ammunition Count Display Evaluation}

Participants will play a FPS level given only one ammo clip, but a large number of enemies to destroy necessitating ammo conservation. We will compare an icon bar (nondiagetic), regular bar (non-diagetic), numerical indicator (nondiagetic), numerical indicator (diagetic, like Dead Space), textual indicator (non-diagetic) and a visualization of remaining bullets (diagetic, as in Metro 2033). Number of enemies destroyed will be used as a metric of performance.

\section{Navigation Aids Display Evaluation}

This task will use a maze that participants will navigate. Mini-map (non-diagetic), directional arrow (semi-diagetic), and no navigation aid will be compared. Completion time will be recorded.

\section{POST-EXPERIMENT}

In the long-term, results of this experiment will inform development of a software tool for semi-automatic generation of FPS UIs. Designers would input the information that they would like to display, along with screen size. The software would output an ideal layout, deciding what should be shown in-game and what would be displayed as an overlay. Further research will focus on proper sizing of interface elements.

\section{REFERENCES}

[1] E. Fagerholt and M. Lorentzon, "Beyond the HUD - User Interfaces for Increased Player Immersion in FPS Games," M.S. thesis, Dept. Comp. Sci and Eng., Chalmers Univ. of Tech., Göteborg, Sweden, 2009.

[2] S. C. Llanos and K. Jørgensen, "Do players prefer integrated user interfaces? A qualitative study of game UI design issues," Proceedings of DiGRA 2011 Conference: Think Design Play, Hilversum, the Netherlands, 2011.

[3] S. Fragoso, "Interface design strategies and disruptions of gameplay: Notes from a qualitative study with first-person gamers," HumanComputer Interaction, Part III, HCII 2014, LNCS 8512, 2014.

[4] V. Zammitto, "Visualization techniques in video games," Proceedings of Electronic Information, the Visual Arts, and Beyond, London, UK, 2008, pp. 267-276.

[5] R. P. McMahan et al, "Considerations for the use of commercial video games in controlled experiments," Entertainment Computing, vol. 2, no. 1, pp. 3-9, March 2011. 